\title{
A Critical Assessment of Epidemiology Studies Regarding Dietary/Supple- mental Zinc and Prostate Cancer Risk
}

\author{
Leslie C. Costello, ${ }^{*}$, Renty B. Franklin ${ }^{1}$ and Ming T. Tan ${ }^{2}$ \\ ${ }^{I}$ Department of Biomedical Sciences/Dental School, and The Marlene and Stewart Greenebaum Cancer Center, Univer- \\ sity of Maryland, 650 West Baltimore Street, Baltimore, Maryland 21201, USA \\ ${ }^{2}$ Department of Epidemiology and Preventive Medicine and Division of Biostatistics, Marlene and Stewart Greenebaum \\ Cancer Center, University of Maryland School of Medicine, 10 South Pine Street, Baltimore, Maryland 21201, USA
}

\begin{abstract}
Despite the prevalence of prostate cancer, the etiology and factors associated with its development and progression are largely unknown. An important relationship in prostate cancer is the role of zinc. Clinical evidence and experimental evidence have established that prostate cancer is associated with a decrease in the zinc uptake and accumulation in the malignant cells; and that the accumulation of zinc in the prostate cells prevents malignancy. In contrast to this established consistent clinical relationship, numerous epidemiology studies and reports of the effect of dietary and supplemental zinc on the incidence of prostate cancer have provided divergent, inconsistent, and inconclusive results; which range from adverse effects of zinc, protective effects of zinc, and no effect of zinc on the risk of prostate cancer. Despite these divergent and inconclusive results, a prevailing view and public warning has evolved from unsubstantiated and uncorroborated epidemiology studies that zinc consumption increases the risk of developing advanced stage prostate cancer. Such a conclusion is not well-founded and has serious, confusing and erroneous implications for the medical/scientific community and for the public-at-large. The admonition of Dimitrios Trichopoulos over a decade ago [1] that, "... (epidemiology) studies will inevitably generate false positive and false negative results with disturbing frequency. ..., when (people) do take us seriously, we may unintentionally do more harm than good" can be applied to the situation that is the subject of this report.

Therefore it is extremely important to review the epidemiology studies that have lead to the conclusion of an adverse effect of zinc, and also that have produced such inconsistent and divergent results. This critical review defines issues, problems, and shortcomings that exist in the conduct, conclusions, and dissemination of the epidemiology studies. We caution that one should be knowledgeable and understanding of these issues in assessing the validity and the conclusiveness of the outcomes from the epidemiology studies of purported associations of dietary and supplemental zinc on the risk of prostate cancer; particularly when the unsubstantiated conclusions are at odds with clinical and experimental evidence. It is in the interest of the medical, scientific and public communities that this critical review is undertaken. We hope that this review will generate an open, objective, scientific and medical discussion and assessment of this important issue.
\end{abstract}

\section{INTRODUCTION}

HealthDay News (July 2, 2003) reported, "Men who overdose on zinc supplements more than double their risk of prostate cancer, a government study finds". The Washington Post (July 1, 2003), "Study Links Zinc, Prostate CancerMen who take too much zinc may be raising their prostate cancer risk, U.S. researchers said yesterday". Moyad [2] advises patients that "larger intakes... (100 mg/day zinc)... should be absolutely discouraged...until adequate research resolves this controversial issue". The Mayo Clinic Health Letter (May, 2004) cites "Large doses of zinc may increase risk of prostate cancer". These and other such pronouncements, while of good intent, convey a public warning that is interpreted as "Caution: zinc is linked to prostate cancer!"; which will have unintended consequences.

*Address correspondence to this author at the Department of Biomedical Sciences/Dental School, and The Marlene and Stewart Greenebaum Cancer Center, University of Maryland, 650 West Baltimore Street, Baltimore, Maryland 21201, USA; E-mail: lcostello@umaryland.edu
The basis for these pronouncements was the epidemiologic report [3], in which the authors state, "...we found that excessively high supplemental zinc intake was associated with an increased risk of advanced prostate cancer". The fact that the report emanated from highly recognized medical institutions (NCI and Harvard) and was published in a major medical/scientific journal provided "automatic acceptance and credibility". Notwithstanding the authors' recognition that further studies are necessary, the power of focus on adverse effects of zinc has had its obvious consequences as evidenced by the above pronouncements. In contrast, an earlier epidemiological report by Kristal et al. [4] had concluded that the daily use of zinc supplement was associated with a significant decrease in prostate cancer. However, one cannot find any public pronouncements that proclaim "studies show that zinc will prevent prostate cancer!" Indeed, much to their credit, Kristal et al. cautioned "...it is important to refrain from making public health recommendations for supplement use for prevention of prostate cancer until there is a much broader and more compelling consensus of evidence". To add to the confusion, other epidemiologic studies report no correlation of dietary/supplemental 
zinc with prostate cancer risk [for reviews see ref 5,6]. The divergent, inconsistent, and inconclusive observations represented in the epidemiologic studies are in sharp contrast to the established clinical relationship that prostate cancer is virtually always associated with a decrease in zinc content of malignant cells and tissue, and is also in contrast to the overwhelming experimental evidence that zinc is a tumorsuppressor agent in prostate cancer [for reviews see 7-9].

So, why all the hype about an adverse effect of zinc? Obviously, the pronouncements are intended to protect the public from a possible cancer causing condition. However, the basis for such "warnings" is not founded on clinical and scientific evidence. It is not based on any consistent and validated epidemiology observations. It is based on the sensationalism that accompanies a suggestion of an impending health crisis. Unfortunately, it also leads to unintended consequences that result in a disservice to the interest of the public-at-large and the medical/scientific community. The warning of zinc association with increased risk of prostate cancer is likely to result in the abandonment of zinc supplemental use by the elderly male population, which is predisposed to decreased bioavailability of zinc and associated health consequences. Moreover, such information also impacts the thinking of the medical and scientific community. More often than not, clinicians and medical researchers will read only the abstract of a published report, or will read the full epidemiologic report without any in depth analysis of the content and validity of the study or the conclusions expressed in the report. From this, future research and direction are formulated, and decisions concerning the funding of research projects are decided on the basis of the speculative, unsubstantiated, and often inaccurate reports. Indeed, some of the very concerns that we raise were recently emphasized by Boffetta et al. [10] who warned of the impact that results from false-positive epidemiological reports, "Increased epistemological humility regarding findings in epidemiology would go a long way to diminishing the detrimental effects of false-positive results on the allocation of limited research resources, on the advancement of knowledge of the causes and prevention of cancer ...".

Consequently, the serious issues surrounding the epidemiology studies and reports dealing with zinc and prostate cancer cannot remain unnoticed, unidentified and unchallenged. Inconclusive and uncorroborated studies that have high impact on public health issues should be carefully and critically reviewed and screened before dissemination through mainstream medical/scientific journals, which carry the implication of validation by virtue of their being published. That safeguard has failed. Therefore we have the responsibility and obligation as scientists to identify and to bring the issues to light; to review critically the conduct of the studies and the presentation and treatment of the data; to question the validity of conclusions and interpretations derived from any flawed and/or unsubstantiated reports; and to provide a clinically and scientifically credible basis in support of our position. We do so with the recognition of the reality that was expressed in Taubes report [1] that "The first one or two papers about a suspected association 'spring into the general public consciousness in a way that does not happen in any other field of scientific endeavor, 'says Harvard's
Walker. And once a possible link is in the public eye, it can be virtually impossible to discredit".

Before proceeding further, we must also provide the following disclosure. We have been and continue to be engaged in clinical and experimental research concerning the role of zinc in prostate cancer. We have published numerous reports and have formulated concepts regarding this issue. We take no position regarding the question of the effect, if any, of dietary/supplement zinc on the development or progression of prostate cancer. We view that the existing epidemiology information to be insufficient and inconclusive; and that associations of dietary/supplement zinc with prostate cancer have not been established to the point that warrants unjudicious public announcements. We do take a strong position that clinical and experimental evidence demonstrate an important role of zinc as a tumor-suppressor agent in prostate cancer, which is a different issue than the effect of dietary/supplement zinc. The latter involves complex multivariate factors in addition to or other than zinc itself. Notwithstanding any perceived or potential "bias", we are confident that this review will stand the test of scientific objectivity and credibility.

\section{THE CLINICAL AND EXPERIMENTAL EVI- DENCE OF THE ROLE OF ZINC IN PROSTATE CANCER}

To address the issues of zinc and prostate cancer, three lines of evidence should be considered: 1) clinical evidence; 2) experimental evidence; 3) epidemiologic evidence. In recent reports [7-9] we have reviewed and detailed the clinical and experimental evidence and background that describe the role of zinc in the development and progression of prostate cancer. In this presentation, we will focus predominantly on the epidemiology studies and evidence. In order for the reader to assess the evidence and the criticisms that we will present, the reader must have a substantial understanding of the clinical and experimental evidence regarding zinc and prostate cancer. We cannot, within the limits of this presentation, reiterate the extensive clinical and experimental evidence; and also present the in-depth analysis of the epidemiology studies. We hope that the reader will avail himself/herself of our extensive reviews for important background information; and will apply that information in concert with the assimilation of the present epidemiology discussions. The following will provide a condensed summary to highlight the clinical and experimental evidence before engaging in the evaluation of the epidemiology studies.

The major anatomical/functional component of the prostate gland is the peripheral zone, which is also the major region where cancer develops and progresses. The peripheral zone glandular epithelial cells have evolved as highly specialized zinc accumulating cells. As a result the normal prostate gland contains about $3 \mathrm{mM}$ zinc, which is 10-15-fold higher than the levels found in other tissues (Table 1). The ability of normal prostate cells to accumulate zinc is due to the expression of ZIP1 zinc uptake transporter. The functional role of zinc accumulation is to inhibit citrate oxidation, which results in the accumulation of extraordinarily high levels of citrate for secretion into the prostatic fluid (Table 1). 
Table 1. Typical Citrate and Zinc Levels (mM Concentration)

\begin{tabular}{|l|c|c|}
\hline & CIT & ZINC \\
\hline \hline NORM PZ & 13 & 3 \\
\hline PCA PZ & 1 & 0.6 \\
\hline OTHER TISSUES & 0.3 & 0.2 \\
\hline NORM PR FLUID & 90 & 9 \\
\hline PCA PR FLUID & 5 & 0.5 \\
\hline BLOOD PLASMA & 0.1 & 0.015 \\
\hline
\end{tabular}

In contrast to the normal prostate gland, prostate cancer is characterized by a marked decrease in zinc levels and in citrate levels (Table 1). Since the first report of Mawson and Fisher in 1952 [11], there have been about 17 published studies that compared the zinc content in resected tissues from cancerous and non-cancerous glands, all of which consistently show a major and significant decrease $(68 \%$ decrease; $\mathrm{P}<0.001)$ ) in zinc levels in prostate cancer. Moreover, the direct analyses of the in situ cellular levels of zinc consistently show that the high zinc level found in normal peripheral zone glandular epithelial cells is markedly depleted in the adjacent malignant cells [12-16]. More revealing information is derived from the zinc analysis of individual subjects. Fig. (1) $[17,18]$ shows that the malignant prostate zinc levels are always low, and one never finds malignant tissue that exhibits the high zinc levels that characterize normal prostate glands. Identical results are obtained with citrate levels which reflect the zinc change that is responsible for the citrate level. In concurrence with the decrease in zinc in malignant cells, ZIP1 expression is also down regulated in the malignant cells. The absence of ZIP1 transporter prevents the uptake and accumulation of high zinc levels in malignant cells. Thus, the consistent and overwhelming clinical evidence irrefutably establishes that, in prostate cancer, the malignant prostate cells in situ lose the ability to accumulate zinc; and that high zinc accumulation is incompatible with malignancy. Fig. (2) illustrates the ZIP1/zinc/citrate relationship in normal and malignant cells in prostate cancer.

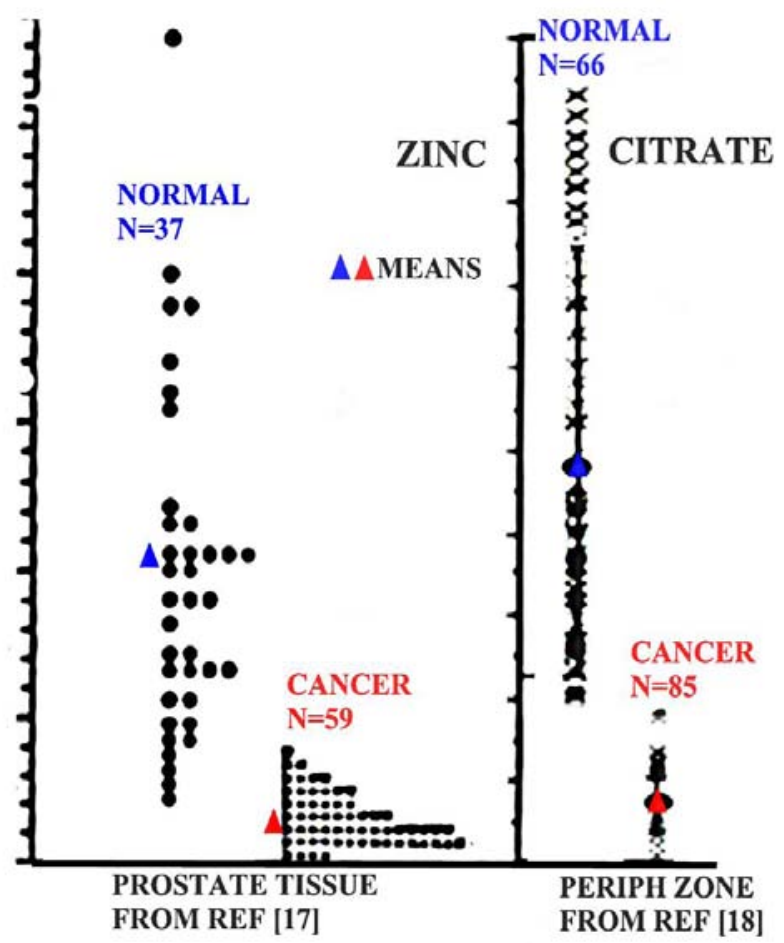

Fig. (1). The comparison of the zinc and citrate levels in normal prostate tissue versus prostate cancer.

Why is zinc accumulation incompatible with the development and progression of malignancy? In the normal glandular epithelial cells, the accumulation of zinc provides an essential functional role relating to citrate production as we described. However the high cellular levels of zinc also imposes potential adverse consequences on prostate cells. Since the normal cells evolved for this function, they have adaptive mechanisms that protect against any adverse effects of zinc. The potential adverse effects of zinc are: 1) the metabolic/bioenergetic effects of inhibition of citrate oxidation and terminal oxidation; 2) the apoptogenic and growth in-

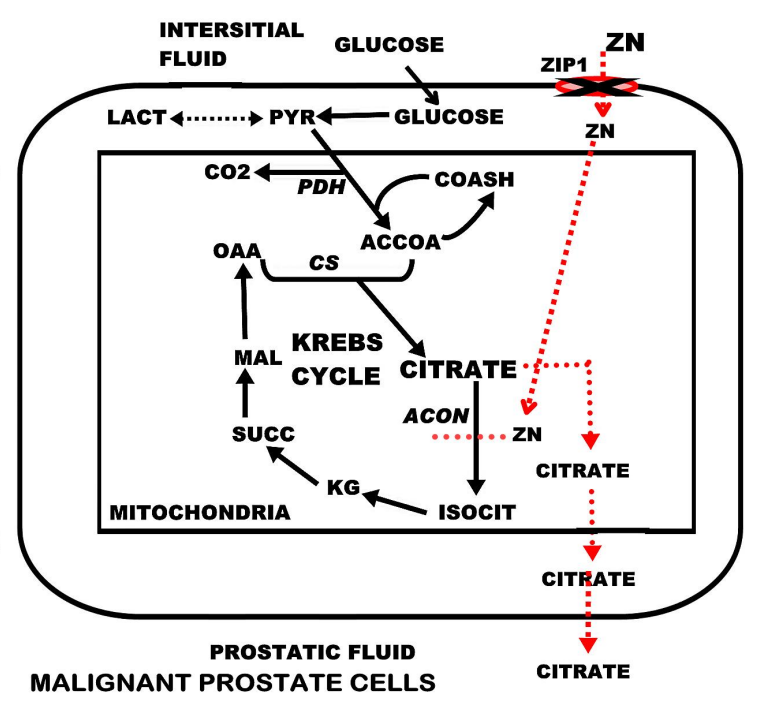

Fig. (2). The comparison of the Zip1, zinc, citrate relationship in normal versus malignant peripheral zone glandular epithelial cells. 
hibitory effects of zinc; and 3) the inhibitory effects of zinc on cell migration and invasion. These are tumor suppressor effects of zinc. The malignant cells avoid these adverse effects of zinc by the down regulation of ZIP1 transporter, which prevents the uptake and accumulation of zinc.

The clinical and experimental evidence and relationships argue against any direct effect of zinc on the prostate gland that would promote or enhance the development and progression of prostate cancer. Any claims that dietary/supplemental zinc promotes prostate cancer through increased prostate zinc levels are in contradiction of the clinical observations and evidence. In the absence of credible evidence for a direct effect of zinc, a number of indirect effects have been invoked. For example, attempts are made to link the ingestion of zinc to prostate cancer via systemic effects of zinc on Insulin-like growth factor, on testosterone levels, on immune responses, and other such interactions; none of which is an established zinc/prostate cancer factor. We have addressed and critiqued the reports involved in these claims and essentially dismissed the validity of these explanations for purported zinc promotion of prostate cancer [19-22].

\section{ZINC SUPPLEMENT VERSUS SUPPLEMENTAL ZINC}

It is important to define the relationship that is being addressed in epidemiologic studies. One must distinguish effects due to "zinc supplements" from effects due to "supplemental zinc". The former includes any and all of the components that are found in the composition of zinc supplements. One must consider that any effects of "zinc supplement" could be due to the zinc component or due to non-zinc components or a combination of both. "Supplemental zinc" effects infer that the zinc component of the supplement is responsible for the associated effect. Epidemiologic studies should carefully define the agent(s) associated with any effects of the use of zinc supplements. If a study involves the effects or associations of zinc supplement use on the incidence of prostate cancer, that study does not identify an association between supplemental zinc and prostate cancer. To arrive at any definitive effect of zinc, effects of other constituents of the supplement must be identified and eliminated. Even the form of the zinc complex (such as histidine, gluconate) as well as contaminating constituents must be considered as potential factors in any effect of the supplement. The reality is that, too often, this important distinction has not been made in published reports and in public pronouncements, and "zinc supplement" use is equated to "supplemental zinc" effects.

\section{INGESTED ZINC VERSUS BIOAVAILABLE ZINC}

Any perceived effects of dietary and/or supplemental zinc on prostate cancer must consider the bioavailability of zinc. Ingested zinc will have no direct effect on prostate cancer unless it is processed into bioavailable zinc. In this sense, bioavailable zinc is defined as gastrointestinal zinc that is absorbed and transported to circulation where it will have cellular and physiological effects. An exception is that zinc in the gut could influence other intestinal components or factors; which, in turn, might have effects on prostate cancer. The complexities and confounding influences of numerous interacting factors that impact the intestinal absorption of zinc are largely unknown, unidentified, and unresolved; and are beyond the scope of this discussion. We have addressed some of these factors in earlier reports [19-22]. For this presentation, some considerations will be highlighted. There exist homeostatic mechanisms that retain a "normal" level of zinc in the body. The absorption/assimilation of intestinal zinc is dependent upon the intestinal content of zinc. As the level of intestinal zinc is increased, the fractional absorption of zinc is decreased. One cannot presume that an increase in dietary and/or supplemental zinc results in a corresponding proportional increase in bioavailable zinc. In addition, the absorption of zinc is dependent upon the composition of the intestinal luminal content. For example high phytate content prevents zinc absorption. Calcium in combination with phytate further decreases zinc absorption. Such known factors and unknown factors make it virtually impossible to predict the level of bioavailable zinc that arises from differing dietary/supplemental conditions.

The only solution to this dilemma is to determine the bioavailable level of zinc. Otherwise, one is dealing with guesswork. Most epidemiologic studies have not included any determinations of bioavailable zinc. It is noteworthy that Platz et al. [23] did attempt to relate bioavailability of zinc (determined by toenail zinc content) to prostate cancer. They showed a statistical insignificant trend for the association of high zinc levels with decrease in prostate cancer; and concluded that, "Moderate and higher zinc concentrations in the range observed in these men appear to be associated with a slightly lower risk of prostate cancer". In a large clinical trial study, Hercberg et al. [24] reported that subjects taking a single daily capsule (containing $120 \mathrm{mg}$ of ascorbic acid, 30 $\mathrm{mg}$ of vitamin E, $6 \mathrm{mg}$ of beta carotene, $100 \mu \mathrm{g}$ of selenium, and $20 \mathrm{mg}$ of zinc) showed a significant increase in plasma zinc after two years; but no change in plasma zinc after seven years.

\section{EFFECTS OF SUPPLEMENTAL ZINC VERSUS DIETARY ZINC}

Some epidemiology reports have distinguished effects of zinc derived from food sources (we refer to as "dietary zinc") versus effects of zinc supplements. Leitzmann et al. [3] state that, in contrast to the adverse effects of zinc supplement, “... zinc obtained from food sources was not associated with prostate cancer risk..." Kolonel [25] similarly reported the absence of an effect by zinc from food sources. This raises the critical question, "Why does zinc supplement result in effects that do not exist with zinc derived from food sources?" Let us first assume that the supplement factor nvolved is zinc. The next issue is the bioavailability of zinc, which requires intestinal zinc absorption and transport into circulation. In normal diet, ingested proteins provide the major source of zinc. During the digestion process, proteolysis converts proteins to amino acids, which are the major zinc ligands that provide zinc for absorption and assimilation. In supplements, zinc exists in a variety of forms; most commonly as ZnGluconate; ZnHistidine; ZnAminoAcid Chelate; ZnAcetate; ZnSulfate, ZnPicolinate, ZnCitrate, and plant extracts as "natural" zinc complexes. However, once ingested, the ligand form of zinc is subject to change. For example, it is unlikely that zinc consumed as ZnAcetate or ZnSulfate exists in that form in the intestine. More likely it is bound to available ligands (e.g. amino acids) that have mod- 
erate to high zinc-binding affinity. Under such consideration, is there any reason to expect that, given the same intestinal load of zinc, zinc from supplements become more bioavailable than normal dietary zinc? It seems unlikely that the intestinal process can discriminate the same zinc ligands derived from food sources from those derived from supplements. Possibly some of the zinc supplement ligands are better assimilated than normal dietary zinc. However there is no evidence of a selective form of supplemental zinc being associated with the purported effects of supplemental zinc.

Alternatively, zinc might not be the agent associated with the adverse effects of zinc supplement. Indeed, Krone and Harms [26] suggested that cadmium contaminant in zinc supplements could be associated with any promotion of prostate cancer. They calculated that, at the high zinc supplement levels reported in the Leitzmann et al. [3] study, the cadmium dose would be as much as $\sim 19$ ug/day. Ingested cadmium accumulates in tissues over time, and we have shown that cadmium is an inhibitor of zinc uptake transporter in prostate cells [27]. Inhibition of zinc uptake transporter is beneficial to the development and progression of prostate malignancy. It is possible that other possible contaminants might contribute to such adverse effects as we have discussed (19). Such a relationship could account for the purported adverse effect of long term use of high levels of zinc supplement as reported by Leitzmann et al. [3]. This possibility was offered in a pursuant report by Lawson et al. [28] who state, "The apparent adverse effect of multivitamin supplements in combination with supplemental zinc on prostate cancer risk could be due to nonessential, potentially harmful trace elements contained in zinc supplements...". It is also conceivable that ingestion of high levels of supplements provide factors that impede or inhibit the intestinal absorption of zinc, so that a decrease in zinc bioavailability occurs. If the level of bioavailable zinc is not ascertained, the interpretation of purported effects of zinc supplement on prostate cancer is speculative.

Furthermore, conflicting epidemiology reports exist regarding the effects of dietary zinc on prostate cancer. Gallus et al. [29] reported "a direct association between high zinc intake and prostate cancer risk, particularly for advanced cancers". Compared to a low zinc intake group of $<9.93$ $\mathrm{mg} /$ day, increased zinc consumption up to a $>15.65 \mathrm{mg} / \mathrm{day}$ group purportedly resulted in a "...significant trend in risk ( $p$ $=0.04) \ldots$ for advanced cancers only...". We addressed a number of concerns regarding the validity of the study and the conclusions of that report in our published comment [22] to which we refer the reader. Obviously all other studies of zinc supplement use will have exceeded (supplement plus dietary) the zinc intake of $16 \mathrm{mg} / \mathrm{day}$; but none report effects similar to those claimed by Gallus et al.

\section{DATA ANALYSIS ISSUES ASSOCIATED WITH EPIDEMIOLOGIC STUDIES}

Despite the absence of any consistent and substantiated evidence and conclusion regarding the issue of zinc dietary/supplement usage and prostate cancer, the adverse effect reported by Leitzmann et al. [3] has had the major impact on the medical/scientific/public-interest community as we already noted. The study reports that, based on the Relative Risk analyses, the high zinc supplement had no effect on the total number of cases of prostate cancer, which was comprised of organ-confined (early) cancer and advanced cancer groups. The study further reports that high zinc supplement use results in a significant increase in the number of cases of advanced prostate cancer, but had no effect on the number of cases of organ-confined prostate cancer. This is a scenario that is not a possible natural event. If one accepts that a significantly increased incidence of advanced cancer does occur with no difference in the incidence of total cancer; a significant decrease in organ-confined cancer must also exist. In the absence of the recognition and explanation for this disparity by the authors, one is lead to conclude that the problem resides in the design of the experiment, and or the management of the data. One issue is the large number of cases (from $28 \%$ to $33 \%$ of the total cases in each group) that have been selectively omitted from the data (Table $\mathbf{2}$ of the Leitzmann report). It is reasonable to explore the possibility that the exclusion of the "missing cases" could result in an overstated advance prostate incidence or an understated organconfined incidence or a combination of both.

The authors do state... "The sum of organ-confined prostate cancer cases and advanced prostate cancer cases does not equal the number of total prostate cancer cases because data on stage was not available for all cases and because we excluded stage T3a cancers in the organ-confined and the advanced categories because they are neither organ-confined nor are they usually advanced and hence do not fall into either group". The arbitrary imposition of criteria and the omission of relevant clinical conditions contribute to the imperfections of the data presentation, and introduce potential bias in the design and validity of the study and the pursuant interpretations. Moreover, it becomes difficult for the reader to assess the validity of the statistical treatment of the data and the conclusions of the authors. Are the statistics derived from the total number of prostate cancer cases presented in their table; or are the statistics derived from the total number of cases in the advanced and organ-confined subgroups? This is important because the statistical significance will be markedly altered if the sum of the subgroups is used as the total cases for comparison rather than the total of all cases, which exceeds the sum of the subgroups being compared. Are the same missing cases uniformly or proportionally eliminated in all subgroups? How are the missing cases categorically assigned in the statistical analyses for organ-confined and advanced cancer incidence? Such issues and shortcomings introduce significant questions relating to the conduct, analyses, and conclusions of the study. Even setting these issues aside, is it not reasonable to ask why these missing cases were included in the reported study if they were to be selectively eliminated from the analyses?

The results of the study were obtained using the Cox model to estimate the relative risk by adjusting other factors. The inference is valid if the sample size is large. In such analysis, the effective sample size is the number of events, i.e., the number of advanced prostate cancer cases instead of the seemingly large total number of patients surveyed. For example, there are only 10 advanced cancer cases in the $>101 \mathrm{mg}$ /day high dose group and 11 cases in the 75-100 $\mathrm{mg} /$ day group. So the findings are based on an extremely small sample size. 
The major reported finding of Leitzmann et al. is stated as "However, compared with nonusers, men who consumed more than $100 \mathrm{mg} /$ day of supplemental zinc had a relative risk of advanced prostate cancer of 2.29 (95\% confidence interval $=1.06$ to 4.95; Ptrend $=.003$ ), and men who took supplemental zinc for 10 or more years had a relative risk of $2.37(95 \%$ confidence interval $=1.42$ to 3.95 ; Ptrend <.001)". Their conclusion seems to be based on the trend analysis of all groups which includes the low-zinc user group. This group exhibits a decrease in the incidence of advanced prostate cancer compared to the non-user group. A trend that provides information about the differences among all groups does not provide the statistical comparison of each zinc-user group with the nonuser group. The statistical practice is to have the trend test among all the groups first. If it is significant, then a pairwise comparison should be performed to establish the precise groups that provide the statistical significance relative to the control group (i.e. the non-user group). It is not clear if such a pairwise analysis was employed. Their presented data appear to show that their major finding is principally due to the comparison of the >101 group with the 1-24 low zinc user group; the latter producing a lower incidence of advanced cancer cases than the non-user group. To explore this possibility, we performed paired analyses of their data based on the numbers of cases that are provided in the Leitzmann et al. report. We attempted to perform the analyses using the total number of cases and also the sum of the advanced and organ-confined cases since the two totals are different. Table $\mathbf{2}$ demonstrates that the high significance results from the comparison of the >101 zinc user group with the 1-24 zinc user group; and the significance is markedly lower when the $>101$ zinc user group is compared with the non-user group (i.e. control group). This is due to the fact that the 1-24 zinc user group exhibits a decrease in the incidence of cancer cases when compared to the non-user group. Based on this paired analysis, the authors might have overstated the effect of $>101$ zinc supplement on the incidence of advanced prostate cancer. [Note: The paired analyses p-values are presented only to show the relative relationships as described. The available data and information limited a calculation of the "true" p-values]. In addition, the data for the 1-24 zinc group vs the non-user group indicate a possible decrease in advanced cases in the 1-24 user group; but this was not a statistical significant decrease between these groups (Table 2). However, this possible decrease appears to be corroborated by the reported $R R=0.75$
(95\% Confidence Interval of 0.56 to 0.99 ) obtained for the age-adjusted 1-24 zinc user group vs the non-user referent $\mathrm{RR}=1$; which seemingly could represent a significant decrease in the incidence of advanced cancer cases.

In regard to the cases of organ-confined prostate cancer, the Leitzmann et al. study shows without exception that all seven of the age-adjusted zinc user groups exhibit an $R R<1.0$ $v s$ the non-user group referent=1.0; with one grouping showing a $\mathrm{P}=0.05$. Additional data presented by Leitzmann and Giovannucci [30] showed that nine out of nine zinc-user groups exhibited multivariate RR values $<1.0$ for organconfined cancer compared to the referent value $=1.0$. Therefore, the Leitzmann et al. study presented data that involve sixteen zinc-user groups, all of which show age-adjusted RR values $<1.0$ for organ-confined cancer when compared to the referent group value $=1.0$. The probability of this outcome is $\mathrm{P}<0.0001$. Despite these collective outcomes, there is no indication or suggestion by the authors of any possible protective effects of zinc.

As we discussed above, if a significant increase in advanced cancer is accompanied by no difference in total cancer, there must be a significant decrease in organ-confined cancer. Seemingly this scenario might exist, but the authors appear to have possibly understated the organ-confined results; and possibly overstated the advanced cancer results. We emphasize that this critical analysis is not undertaken or intended to show that zinc supplement has any effect or no effect on prostate cancer risk. We do not know what, if any, effect of zinc supplement might have on prostate cancer. We can only base our concerns and critique on the data and description that are provided in the report; so we recognize that appropriate explanations could alleviate and even correct these concerns. We consider that the Leitzmann et al. report is a potentially important study. Our purpose is to demonstrate the existence of critical issues that exist in the Leitzmann et al. report (and in other epidemiology reports), which militate against the definitive conclusions arising from the study. We believe that these important issues should be addressed and resolved. It might well be that high "zinc supplement" (as distinguished from high "supplemental zinc") will prove to have adverse effects on the development of advanced prostate cancer under some conditions. It might well be that the study could reveal that zinc supplement also exhibits a protective effect against organ-confined prostate cancer. Whatever the study satisfactorily reveals becomes

Table 2. Paired Analysis for Advanced Cancer $p$ Values

\begin{tabular}{|c|c|c|}
\hline 101 zinc group (10/36) vs nonuser group (317/2127) & 0.06 & 0.06 \\
\hline 1-24 zinc group (56/469) vs nonuser group (317/2127) & 0.11 & 0.11 \\
\hline \multicolumn{3}{|l|}{ (no. adv cancer cases/total no. cases) ${ }^{*}$ Fisher exact test; ${ }^{* *}$ Chi-square } \\
\hline 101 zinc group (10/24) vs 1-24 zinc user group (56/338) & 0.005 & 0.005 \\
\hline 1-24 zinc group (56/338) vs nonuser group (317/1540) & 0.10 & 0.11 \\
\hline
\end{tabular}


the acceptable results of the study. Then, the study will have provided significant information and revelations; and will raise important issues and potential relationships that will need to be pursued by future clinical, experimental, and epidemiology investigations.

7. WHAT HAVE EPIDEMIOLOGIC STUDIES ESTABLISHED REGARDING THE ASSOCIATION OF DIETARY/SUPPLEMENTAL ZINC WITH PROSTATE CANCER?

We have not reiterated a summation of all of the epidemiology studies because this has been done by others [5, 6]. There is a split among the studies, which purport to show that dietary/supplemental zinc is associated with protection, or with promotion, or with no effect on prostate cancer; i.e. a virtual absence of any unanimity among the studies. Nearly every report summarizes the study with essentially the same conclusion, "More studies are needed" to establish the issue being addressed. We question if repeating more of the same will only provide more inconsistent and speculative information; and is likely to unravel nothing of consequence that is new. An explanation for the inconsistent results could be that one cannot expect consistency in comparing the results of differing population studies because the conditions in each study might be different. While there is some validity to this explanation, the fact remains that there are overlapping conditions in some of the reports; yet the results and conclusions are different and divergent. For example, the Gallus et al. report [29] essentially states that a person who has a dietary intake of $16 \mathrm{mg}$ zinc/day will more likely develop advanced prostate cancer than a person with a $10 \mathrm{mg} / \mathrm{day}$ zinc intake. Most elderly males have a zinc intake of $\sim 13-14 \mathrm{mg} /$ day. Leitzmann et al. report that over the range of $13 \mathrm{mg} / \mathrm{day}$ (dietary zinc alone) to $38 \mathrm{mg} /$ day (dietary plus supplement), there is no effect on prostate cancer. Kristal et al. [4] report that individuals who have a daily zinc intake of $\sim 20-30$ $\mathrm{mg}$ /day (we estimate $13 \mathrm{mg} /$ day dietary plus $10-20 \mathrm{mg} /$ day supplement) show a decrease in prostate cancer. Can one expect that precise differences of estimated consumption (an oxymoron) of $2 \mathrm{mg} /$ day zinc in diet produce such profound consequences as Gallus et al. claim? Should not such purported implications bear some reasonable relationship to the realities of life? Moreover, the low-dose zinc use of the Leitzmann et al. report likely overlaps with the zinc levels involved in the Kristal et al. report. However, each report draws a different conclusion regarding the effects of supplemental zinc on prostate cancer. What are the reasons for such disparities among these and other studies?

\section{EPIDEMIOLOGISTS IGNORING THEIR OWN ADMONITIONS}

Our concerns and criticisms regarding the epidemiology studies of zinc and prostate cancer are amplified by visiting the critical analyses of epidemiology studies presented in the informative report of Taubes; which includes discussions, comments, and responses of eminent epidemiologists [1, 31]. We urge all readers of our presentation to read also the Taubes report for a full and expanded appreciation of the critical epidemiology issues in general and specifically as they relate to the issue of zinc and prostate.

\section{a. The Issue of Reliability of Relative Risk Analysis}

Pertaining to the application of Relative Risk, Taubes [1] cites the following comments:

Marcia Angell, editor of the New England Journal of Medicine states "As a general rule of thumb, we are looking for a relative risk of three or more [before accepting a paper for publication ]...". Robert Temple, director of drug evaluation at the Food and Drug Administration states "An association is generally considered weak if the odds ratio [relative risk] is under 3.0 and particularly when it is under 2.0...". Sir Richard Doll suggested that "no single epidemiologic study is persuasive by itself unless the lower limit of its $95 \%$ confidence level falls above a threefold increased risk". Other researchers, such as Harvard's Trichopoulos, opt for a four-fold risk increase as the lower limit. Dr. Kabat, IAQC epidemiologist states "My basic rule is if the relative risk isn't at least 3 or 4, forget it". Gori [32] summarizes with, "As other professionals have done, epidemiologists could establish a code of good practice, spelling out optimal standards of hypothesis formulation, study design, and conduct. Structural uncertainties should limit heuristic causal inferences to relative risk or odds ratio values above 3 or 4...".

Additionally, a consensus exists that even these recommended RR values should be viewed with skepticism unless strengthened with corroboration by other epidemiological studies and by a meaningful established clinical/experimental body of evidence. As we have discussed, neither of these criteria exists for the epidemiologic studies relating to adverse effects of zinc on prostate cancer. Now consider the results of virtually all the reports pertaining to zinc ingestion and prostate cancer as represented in the following examples. Leitzmann et al. [3] report RR values of 2.29 and 2.37, which leads to their conclusion of increased risk of prostate cancer. Gallus et al. [29] report OR values of 2.02 and 1.56 from which they conclude that high levels of dietary zinc increases the incidence of prostate cancer. Kolonel et al. [25] report OR value of 1.7 for a zinc intake group and conclude an increase risk of cancer due to zinc. Kristal et al. [4] report an OR value of 0.55 (reciprocal OR value $=1.8$ ) for a supplemental zinc decrease in risk of prostate cancer. Based on the criteria described above, and when combined with those reports of no effect of zinc on relative risk, it is reasonable to conclude from the collective epidemiology studies "ingestion of dietary/supplement (above some unknown minimal daily zinc requirement) has no established significant effect on the relative risk of prostate cancer?"

\section{b. The Issue of Statistical Application}

To circumvent this issue, some epidemiologists resort to statistical verification, particularly the application of the limits of the $95 \%$ confidence interval. To this point, Taubes [1] cites the assessment of Breslow, "... such statistical "confidence" means considerably less than it seems to. The calculation of confidence limits only takes into consideration random variation in the data. It ignores the systematic errors, the biases and confounders, that will almost invariably overwhelm the statistical variation". Gori [32] concludes, 
"Most of the epidemiology of multifactorial diseases fails a test of method, due to absent experimental randomization and unachievable control of biases and confounders". Added to this is the admonition (1): "Bias and confounders are the plague upon the house of epidemiology", says "Philip Cole, chair of epidemiology at the University of Alabama. They represent anything that might lead an epidemiologic study to come up with the wrong answer, to postulate the existence of a causal association that does not exist or vice versa".

These concerns are particularly applicable to the epidemiology studies regarding zinc and prostate cancer. Consider the following representative footnote appended to a table of relative risks of prostate cancer from zinc supplement use: "RR (95\% CI) adjusted for current age, body mass index at age 21, height at baseline in 1986, pack-years of smoking in the previous decade, family history of prostate cancer, vigorous physical activity, regular aspirin use, intake of total energy, dietary calcium, supplemental calcium, fructose, supplemental vitamin E, tomato-based foods, fish, red meat, and a-linolenic acid".; none of which, other than age and possible family history, is an established factor in the natural history of prostate cancer. Such a litany of un-established factors and conditions diminishes the likelihood that they have any significant relevance or impact on the incidence of prostate cancer and its stages of development and progression. There is little, if any, value to the identification of statistical associations that bear no reasonable relationship to an event. The need to justify such associations by extreme speculation of their relationship is evidence of the absence of a plausible meaningful association.

There is no such thing as "good data" and "bad data". The data accurately represent and reflect the outcome of a study based on all the existing known and unknown conditions, factors, and variables for each group that is included in the study and for the study as a whole. Statistical analyses cannot discriminate the data from those studies that are well controlled studies from those studies that are poorly controlled studies. The study is reliable only when an appropriate experimental design is coupled with valid statistical analyses followed by an appropriate interpretation of the experimental results. In the absence of all three ingredients, the study and its conclusions become highly suspect and/or invalid. Breslow was quite accurate in his assessment of the application of mathematical/statistical models [1], "But the question remains: What is the fundamental quality of the data, and to what extent are there biases in the data that cannot be controlled by statistical analysis? One of the dangers of having all these fancy mathematical techniques is people will think they have been able to control for things that are inherently not controllable".

\section{THE IMPACT ON SOCIETY/ WHAT NEEDS TO BE DONE}

We return to the admonition of Dimitrios Trichopoulos, “...(epidemiology) studies will inevitably generate false positive and false negative results with disturbing frequency".; and further adds "We are fast becoming a nuisance to society...People don't take us seriously anymore, and, when they do take us seriously, we may unintentionally do more harm than good". Brian MacMahon cautioned [1, 33], "There would be few drawbacks to publishing weak, uncer- tain associations if epidemiologists operated in a vacuum, but they do not. .... By the time the information reaches the public mind, via print or screen, the tentative suggestion (of association) is likely to be interpreted as a fact...". Added to this is the comment (1), "The first one or two papers about a suspected association 'spring into the general public consciousness in a way that does not happen in any other field of scientific endeavor', says Harvard's Walker. And once a possible link is in the public eye, it can be virtually impossible to discredit". These admonitions are applicable to the concerns that we have raised regarding unsubstantiated and questionable reports that purport to establish an effect of dietary/supplemental zinc and prostate cancer; and that become medical and public "high profile" publications.

Obviously one cannot and should not expect to divorce the publication of any scientific reports from their entry into the public domain. However, in the chain of events, some responsible actions and considerations are available. The first responsibility lies with the investigators of the studies. They must first appreciate the issues, pitfalls and limitations that are inherent in the experimental approach of the study. If they choose to ignore the deficiencies of the study and opt to manipulate the study and data to achieve a desired outcome, they are in violation of their responsibilities. If they disregard or fail to reveal and discuss other relevant epidemiology reports that are in conflict, they are withholding important information from the reader. If they seek out or respond to "the press", they are magnifying the impact and credibility of their study; regardless of any included disclaimer statements of the "tentativeness" of the results. In summary, they need to be aware of the admonitions and the potential impact of their reported studies. The medical/scientific/public interests should be of primary concern.

Secondly, the journals that publish such studies and their editorial boards and reviewers also bear a major responsibility since they are the vehicles for widespread dissemination of the study. The acceptance and publication of flawed papers give credibility to the study. Other than those who have an intimate interest in a published report, a critical assessment of the study is not made by readers of the report. Once a report is in the medical, scientific, and public domain, any further use or interpretation of the information is "fair game', but it does not relieve the investigators and the journals from the responsibility of the impact of publication of inconclusive and/or uncorroborated information. The responsibility is even greater when there is the likelihood that the subject matter will have a public and medical "high-impact"; and increased likelihood of gaining the attention of "the press". Moreover, the journal becomes complicit with the press when the journal issues "press releases" (as has been done for zinc and prostate cancer). Often, it is the "press release" that initiates the widespread entry of the report into the definitive interest and concern of the medical community and the public-at-large. A more responsible and judicious role of the journals is necessary.

The next step is the role and responsibility of the "press" in the chain of events. This is outside of any "control" by the scientific community. There are economic and other interests and priorities that drive the "press" in regard to when and how it will emphasize its reporting. Investigators and journals that engage the public press cannot invoke any claim of 
misrepresentation in the reporting by the press. Why not let the scientific/medical process work its way to reach a substantial body of evidence that solidifies an important relationship; and then provide the important public pronouncements; especially when an immediate and catastrophic issue is not involved?

Then, we reach the "responsibility" of the public. It is foolhardy to set a standard, such as the public needs to know how to assess the medical/scientific information. In fact, most of the people likely to be impacted by these reports, are the least likely to have the background, education, and personal involvement to make any such assessments. They believe what they read. They do not and cannot evaluate the information. They will often pursue additional information from the medical community. However, as we discussed, the medical community is often also "ignorant" of the validity of the claims, and become perpetuators of the misinformation.

Therefore, we place the primary responsibilities on the investigators that provide the report and on the journals that publish the reports. This is the first line of defense.

\section{SUMMARY AND CONCLUSIONS}

Clinical and experimental studies have established that zinc levels are decreased in prostate cancer and support a role of zinc as a tumor suppressor agent. Malignant prostate cells in situ are incapable of accumulating high zinc levels from circulation.

We conclude that there is no established relationship provided by epidemiologic studies for any effect or lack thereof of dietary/supplement zinc on the risk of prostate cancer. Proclamations of an association of dietary/supplemental zinc and increased prostate cancer are based on inconclusive and uncorroborated reports. This remains an unanswered question.

There are important concerns and issues regarding the conduct and outcome of epidemiologic studies regarding the association of dietary/supplement zinc intake on prostate cancer.

It is the responsibility of investigators who generate a report and the medical and scientific journal that publishes the report to insure the credibility of the studies that are accepted for publication, and the same is required of those who agree to serve as reviewers of the manuscripts. It is insufficient and irresponsible to apply a disclaimer that the study being published is the sole responsibility of the authors of the paper.

The scientific and medical community and the public-atlarge should be made cognizant of the limitations and inadequacies of the epidemiologic studies before the acceptance of any unsubstantiated conclusion or proclamation concerning dietary/supplement zinc and prostate cancer; especially when it conflicts with established clinical and experimental evidence.

It is questionable that future epidemiologic studies that employ the same format of previous studies are likely to provide any additional conclusive, consistent, and meaningful information regarding this issue. The issue of any association (harmful or beneficial) of dietary/supplemental zinc will be resolved by much needed well-controlled clinical trials.

We hope that this report will have raised and brought to light the issues surrounding the epidemiologic studies concerning zinc and prostate cancer. They need to be addressed, debated, and ultimately resolved. Epidemiology evidence provides the important third leg (along with clinical and experimental evidence) to provide the health and clinical aspects of the relationship of zinc and prostate cancer as with any other health-associated condition. To do so is in the interest of the medical/scientific community and the public-atlarge.

\section{ADDENDUM}

During the final stage of publication, a new relevant paper by Wagner et al. ${ }^{1}$ came to our attention. The report is a population study of the relationship of soil zinc content, groundwater usage, and prostate cancer incidence. The report concludes "Increased prostate cancer rates were associated with reduced soil zinc concentrations and elevated groundwater use...". Statistically, the report exhibits similar concerns that we have raised for other studies. However, the results provide additional evidence consistent with the relationship that low zinc availability can have an adverse effect by increasing the incidence of prostate cancer. Moreover, the study adds to the necessity for resolution of the issues of the relationships of the ingestion of zinc on the incidence of prostate cancer.

\section{ACKNOWLEDGEMENT}

The studies of LCC and RBF referred to in this paper were supported by NIH grants CA79903; CA71207.

\section{REFERENCES}

[1] Taubes G. Epidemiology faces its limits. Science 1995; 269: 164-9.

[2] Moyad MA. Zinc for prostate disease and other conditions: a little evidence, a lot of hype, and a significant potential problem. Urol Nurs 2004; 24: 49-52.

[3] Leitzmann MF, Stampfer MJ, Wu K, Colditz GA, Willett WC, Giovannucci EL. Zinc supplement use and risk of prostate cancer. J Natl Cancer Inst 2003; 95: 1004-7.

[4] Kristal AR, Stanford JL, Cohen JH, Wicklund K, Patterson RE. Vitamin and mineral supplement use is associated with reduced risk of prostate cancer. Cancer Epidemiol Biomark Prev 1999; 8: 887-92.

[5] Platz EA, Helzlsouer KJ. Selenium, zinc, and prostate. Epidemiol Rev 2001; 23: 93-101.

[6] Silvera SAN, Rohan TE. Trace elements and cancer risk: a review of the epidemiologic evidence. Cancer Causes Cont 2007; 18: 7-27.

[7] Costello LC, Franklin RB. The clinical relevance of the metabolism of prostate cancer; zinc and tumor suppression: connecting the dots. Mol Cancer 2006; 5: 17.

[8] Franklin RB, Costello LC. Zinc as an anti-tumor agent in prostate cancer and in other cancers. Arch Biochem Biophys 2007; 463: 211-17.

[9] Franklin RB, Milon B, Feng P, Costello LC. Zinc and zinc transporters in normal prostate function and the pathogenesis of prostate cancer. Front Biosci 2005; 10: 2230-9.

[10] Boffetta P, McLaughlin JK, La Vecchia C, Tarone RE, Lipworth L, Blot WJ. False-positive results in cancer epidemiology: A plea for epistemological modesty. J Nat Cancer Inst 2008; 100: 988-95.

\footnotetext{
${ }^{1}$ Wagner SE, Burch JB, Hussey J, Temples T, Bolick-Aldrich S, MosleyBroughton C, Liu Y, Hebert JR. Soil zinc content, groundwater usage, and prostate cancer incidence in South Carolina. Cancer Causes Control. 2008 Oct 24. [Epub ahead of print].
} 
[11] Mawson CA, Fischer MI. The occurrence of zinc in the human prostate gland. Can J Med Sci 1952; 30: 336-9.

[12] Hall T. X-ray fluorescence analysis in biology. Science 1961; 18: 449-55.

[13] Schrodt GR, Hall T, Whitmore WF Jr. The concentration of zinc in diseased human prostate glands. Cancer 1964; 17: 1555-66.

[14] Gyorkey F, Min K-W, Huff JA, Gyorkey P. Zinc and magnesium in human prostate gland: Normal, hyperplastic, and neoplastic. Cancer Res 1967; 27: 1349-53.

[15] Vartsky D, Shilstein S, Bercovich A, et al. Prostatic zinc and prostate specific antigen: an experimental evaluation of their combined diagnostic value. J Urol 2003; 170: 2258-62.

[16] Franklin RB, Feng P, Milon BC, et al. hZIP1 zinc uptake transporter down regulation and zinc depletion in prostate cancer. Mol Cancer 2005; 4: 32.

[17] Zaichick VYe, Sviridova TV, Zaichick SV. Zinc in the human prostate gland: normal, hyperplastic and cancerous. Int Urol Nephrol 1997; 29: 565-74.

[18] Kurhanewicz J, Vigneron DB, Hricak H, Narayan P, Carroll P, Nelson SJ. Three dimensional hydrogen-1 MR spectroscopic imaging of the in situ human prostate with $(0.24-0.7-\mathrm{cm} 3)$ high spatial resolution. Radiology 1996; 198: 795-805.

[19] Costello LC, Feng P, Milon B, Tan M, Franklin RB. The role of zinc in the pathogenesis and treatment of prostate cancer: Critical issues to resolve. Prostate Canc Prostate Dis 2004; 7: 111-7.

[20] Costello LC, Franklin RB, Feng P, Tan M, Bagasra O. Zinc and prostate cancer: A critical scientific, medical and public interest issue. Cancer Causes Cont 2005; 16: 901-15.

[21] Costello LC, Franklin RB, Feng P, Tan M. Re: Zinc supplement use and risk of prostate cancer. J Natl Cancer Inst 2004; 96: 23940.
[22] Costello LC, Franklin RB. Re: Dietary zinc and prostate cancer risk: a case-control study from Italy. Eur Urol 2007; 52: 1262-3.

[23] Platz EA, Helzlsouer KJ, Hoffman SC, Morris JS, Baskett CK, Comstock GW. Prediagnostic toenail cadmium and zinc and subsequent prostate cancer risk. Prostate 2002; 52: 288-96.

[24] Hercberg S, Galan P, Preziosi P, et al. The SU.VI.MAX Study: a randomized, placebo-controlled trial of the health effects of antioxidant vitamins and minerals. Arch Intern Med 2004; 164: 233542.

[25] Kolonel LN, Yoshizawa CN, Hankin JH. Diet and prostatic cancer: a case-control study in Hawaii. Am J Epidemiol 1988; 127: 9991012.

[26] Krone CA, Harms LC. Re: zinc supplement use and risk of prostate cancer. J Natl Cancer Inst 2004; 95: 1556.

[27] Guan Z, Kukoyi B, Feng P, et al. Kinetic identification of a mitochondrial zinc uptake transport process in prostate cells. J Inorganic Biochem 2004; 97: 199-206.

[28] Lawson KA, Wright ME, Subar A, et al. Multivitamin use and risk of prostate cancer in the National Institutes of Health-AARP Diet and Health Study. J Natl Cancer Inst 2007; 99: 754-64.

[29] Gallus S, Foschi R, Negri E, et al. Dietary zinc and prostate cancer risk: a case-control study from Italy. Eur Urol 2007; 52: 1052-6.

[30] Chang ET, Hedelin M, Adami H-O, Gronberg H, Bälter KA. Re: Zinc Supplement Use and Risk of Prostate Cancer. J Natl Cancer Inst 2004; 96: 1108-9.

[31] Letters re: The Discipline of Epidemiology. Science 1995; 269: 1325-8.

[32] Gori GB. Letters re: The Discipline of Epidemiology. Science 1995; 269: 1327-8.

[33] MacMahon B. Editorial: Pesticide residues and breast cancer? J Nat Cancer Inst 1994; 86: 572-3. 